\title{
Decimeter-Scale Atomically Thin Graphene Membranes for Gas-Liquid Separation
}

Dandan Hou ${ }^{1,4}$, Shengping Zhang ${ }^{1,2,3,4}$, Xiaobo Chen ${ }^{1}$, Ruiyang Song ${ }^{1}$, Dongxu Zhang ${ }^{4}$, Ayan Yao ${ }^{4}$, Jiayue Sun ${ }^{4}$, Wenxuan Wang ${ }^{4}$, Luzhao Sun ${ }^{3,4}$, Buhang Chen ${ }^{4}$, Zhongfan Liu ${ }^{3,4}$, and Luda Wang $1,2,3,4 *$

${ }^{1}$ Institute of microelectronics, School of Electronics Engineering and Computer Science, Peking University, Beijing, 100871, China.

${ }^{2}$ Academy for Advanced Interdisciplinary Studies, Peking University, Beijing, 100871, China.

${ }^{3}$ Center for Nanochemistry, Beijing Science and Engineering Center for Nanocarbons, Beijing National Laboratory for Molecular Sciences, College of Chemistry and Molecular Engineering, Peking University, Beijing, 100871, China.

${ }^{4}$ Beijing Graphene Institute, Beijing, China, 100095, China.

*Corresponding author: Prof. Luda Wang

E-mail address: luda.wang@ @ku.edu.cn

KEYWORDS: nanoporous atomically thin membranes (NATMs), CVD graphene, nanoscale pores, gas-liquid separation, large area 


\section{List of Contents}

Figure S1 Different adhesion performance of graphene to the substrates.

Figure S2 Schematic of the three-component phase diagram for a polymer/solvent/non-solvent system.

Figure S3 Digital photos of membranes at different times during the two types of phase inversion processes.

Figure S4 Distribution of pore size and open porosity data of PVDF membranes.

Figure S5 SEM images of PVDF membranes prepared by the VIPS method under different temperature and humidity conditions.

Figure S6 Homogeneity characterization of the decimeter-scale polymeric membranes.

Figure S7 SEM images of the nanoporous atomically thin membranes (NATMs).

Figure S8 SEM images of graphene on copper foil.

Figure S9 Diffusion-driven transport data of $\mathrm{KCl}$ through various membranes.

Figure S10 Digital photo of the nanoporous graphene/polymer composite membrane after $\mathrm{CO}_{2}$ transport experiment. 

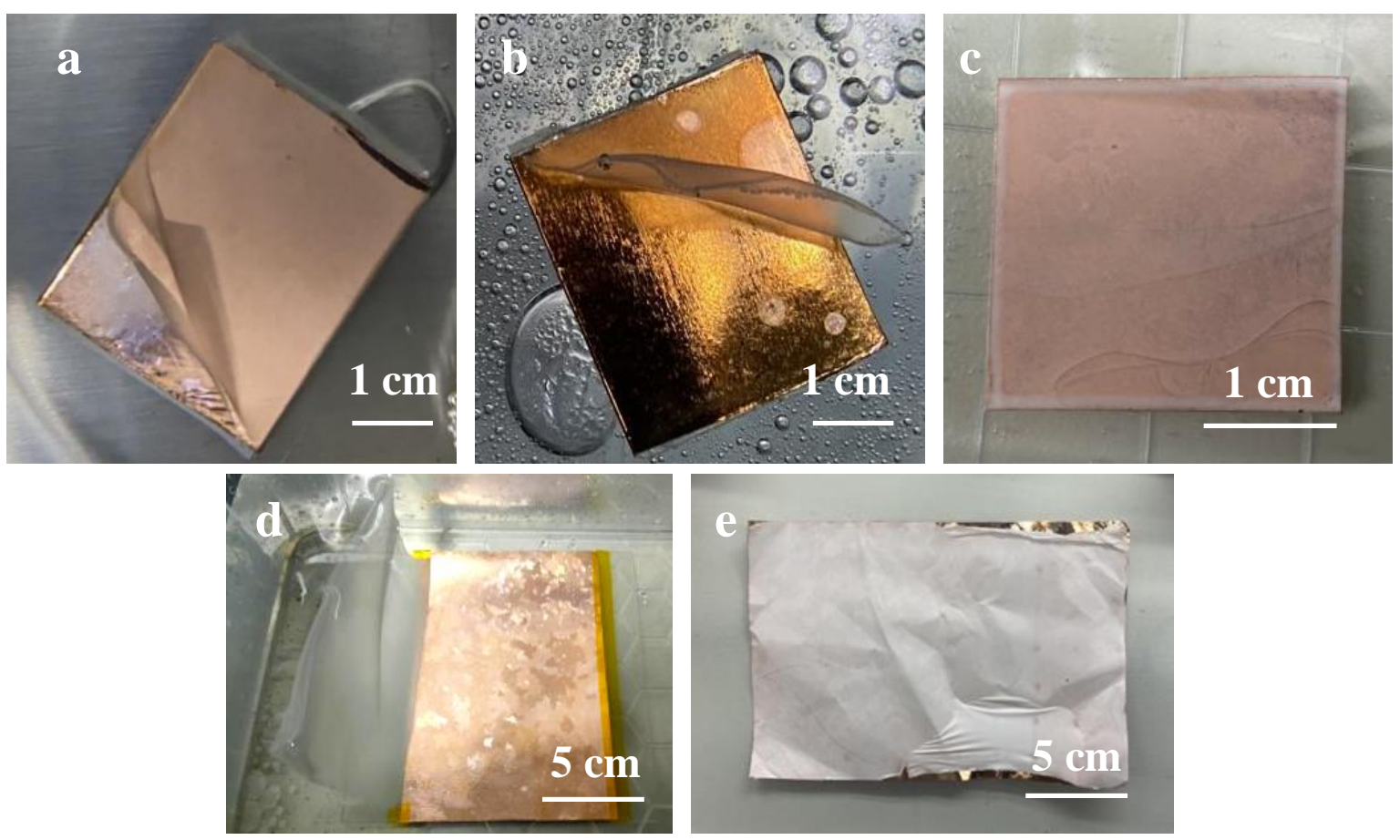

Figure S1 Different adhesion performance of graphene to the substrates. a) Polymer casting directly on the original $\mathrm{Cu}$ foil without any pretreatment. b) Casting on $\mathrm{Cu}$ foil after chemical polishing and annealing. c) Casting on graphene film grown on $\mathrm{Cu}$ foil. d) Non-solvent induced phase inversion of A6-sized $\left(15^{*} 10 \mathrm{~cm}^{2}\right)$ PVDF membrane in a water bath. e) The edges of the membrane crimped and curled after vapor-induced phase inversion at unsuitable humidity and temperature conditions.

When casting on the original $\mathrm{Cu}$ foil or polished and annealed ones, the polymer substrates obviously shrunk and warped during phase inversion process in a water bath, and automatically separated from the $\mathrm{Cu}$ foil. Whereas the polymer substrate kept good adhesion to the surface when casting on the graphene films with an area of $\sim 3 * 3 \mathrm{~cm}^{2}$. When the area of membranes was enlarged in decimeter-scale, many problems arose, such as peeling off automatically during phase inversion in a water bath, or shrinking and crimping in the vapor-induced phase inversion (VIPS) process, resulting in a fail of transfer of graphene onto polymer substrate. These problems were successfully solved by replacing the liquid water with the water vapor environment and adjusting the humidity and temperature parameters during the VIPS process. 


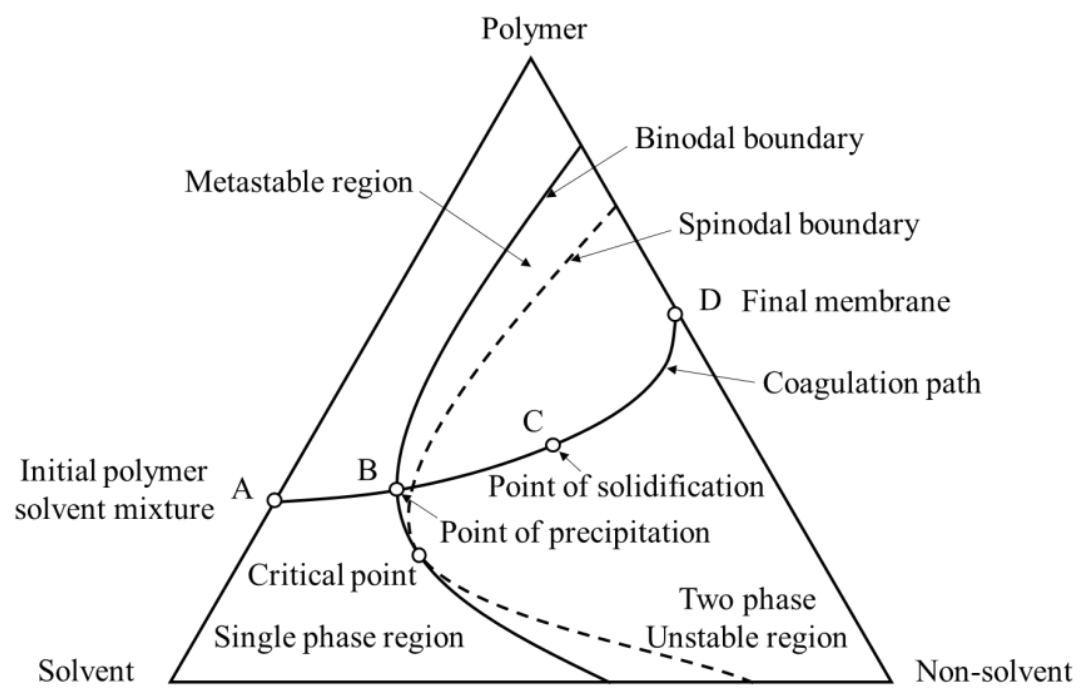

Figure S2 Schematic of the three-component phase diagram for a polymer/solvent/non-solvent system. In the coagulation path, A and D represent the initial polymer solvent mixture and the final membrane formation, respectively. Two phase separation can begin with $\mathrm{B}$. The further movement of the polymer backbone is blocked on $\mathrm{C}$. 

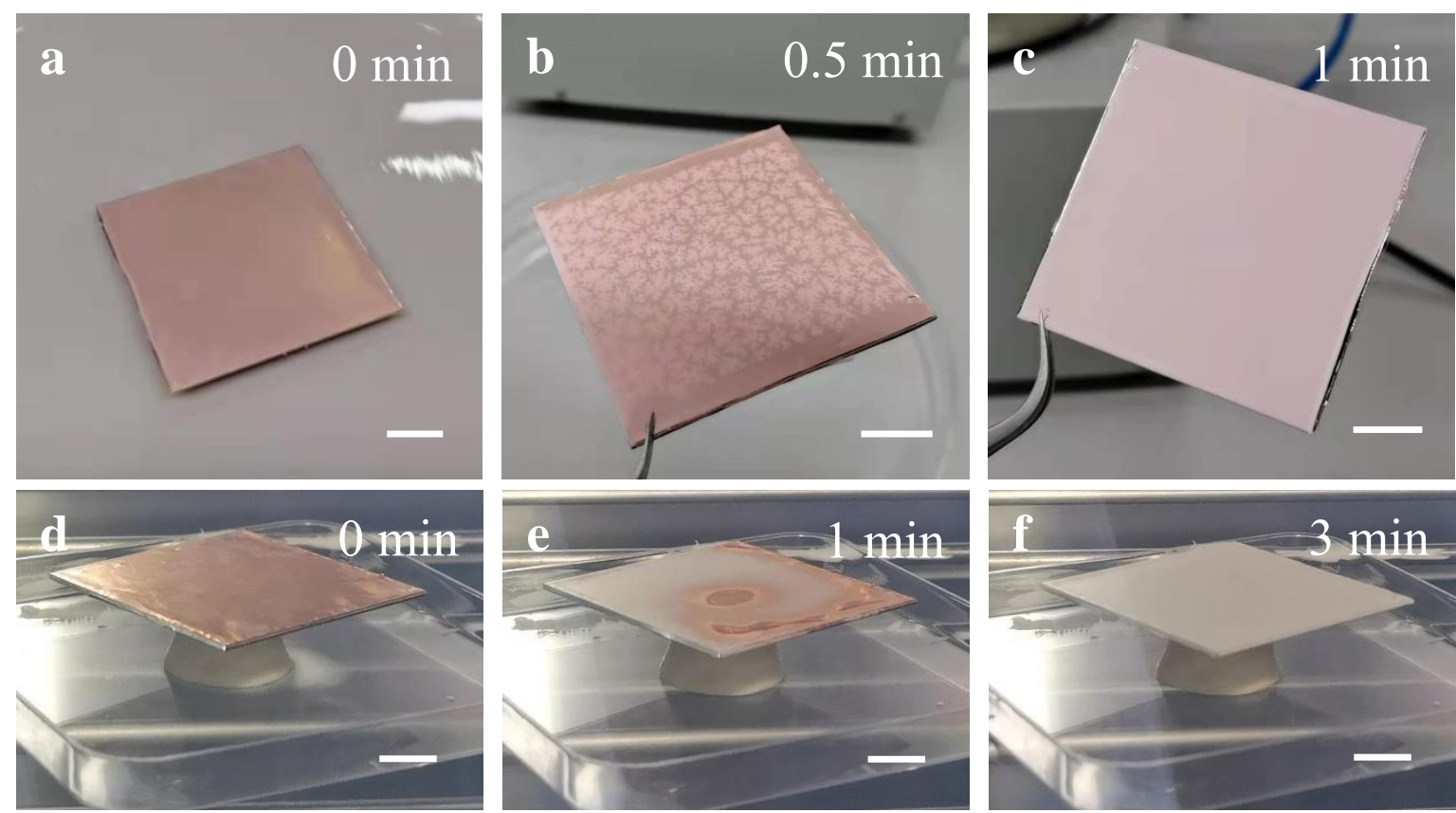

Figure S3 Digital photos of membranes at different times during the two types of phase inversion processes. (a-c) NIPS method (room temperature, $\left.\sim 25^{\circ} \mathrm{C}\right) ;(\mathrm{d}-\mathrm{f})$ VIPS method $\left(60^{\circ} \mathrm{C}\right.$ and $60 \%$ humidity). The areas of the membranes are $\sim 5^{*} 5 \mathrm{~cm}^{2}$. Scale bars represent $1 \mathrm{~cm}$. We can observe obviously the relative slowdown of the coagulation rate of the casting solution during VIPS process when compared with NIPS method. The casting solution/ graphene/copper was immersed in a water bath for tens of seconds for phase conversion (Figure S3a). It is noteworthy that the polymer skeleton has formed and the coagulation process of the casting solution has almost completed within tens of seconds before being taken out of the water bath. During the NIPS process, two phase separation occurred immediately when the casting film was directly immersed into a water bath. The water diffused into the casting film quickly at the initial stage, leading to the severe precipitation of the top layer and the formation of the dense skin layer, which acted as a barrier to the further diffusion of water and reduced the extent and rate of the precipitation in the subsequent process. The membranes will remain this translucent state if not being taken out of the water. Therefore, the digital photos (a-c) mainly illustrate the process of water evaporation. Whereas, the digital photos (d-f) show the whole phase separation process induced by water vapor. For VIPS method, the exchange rate of solvent and non-solvent was reduced dramatically, and the coagulation rate of the casting solution was slowed down, resulting in the successful removal of the skin layer. 

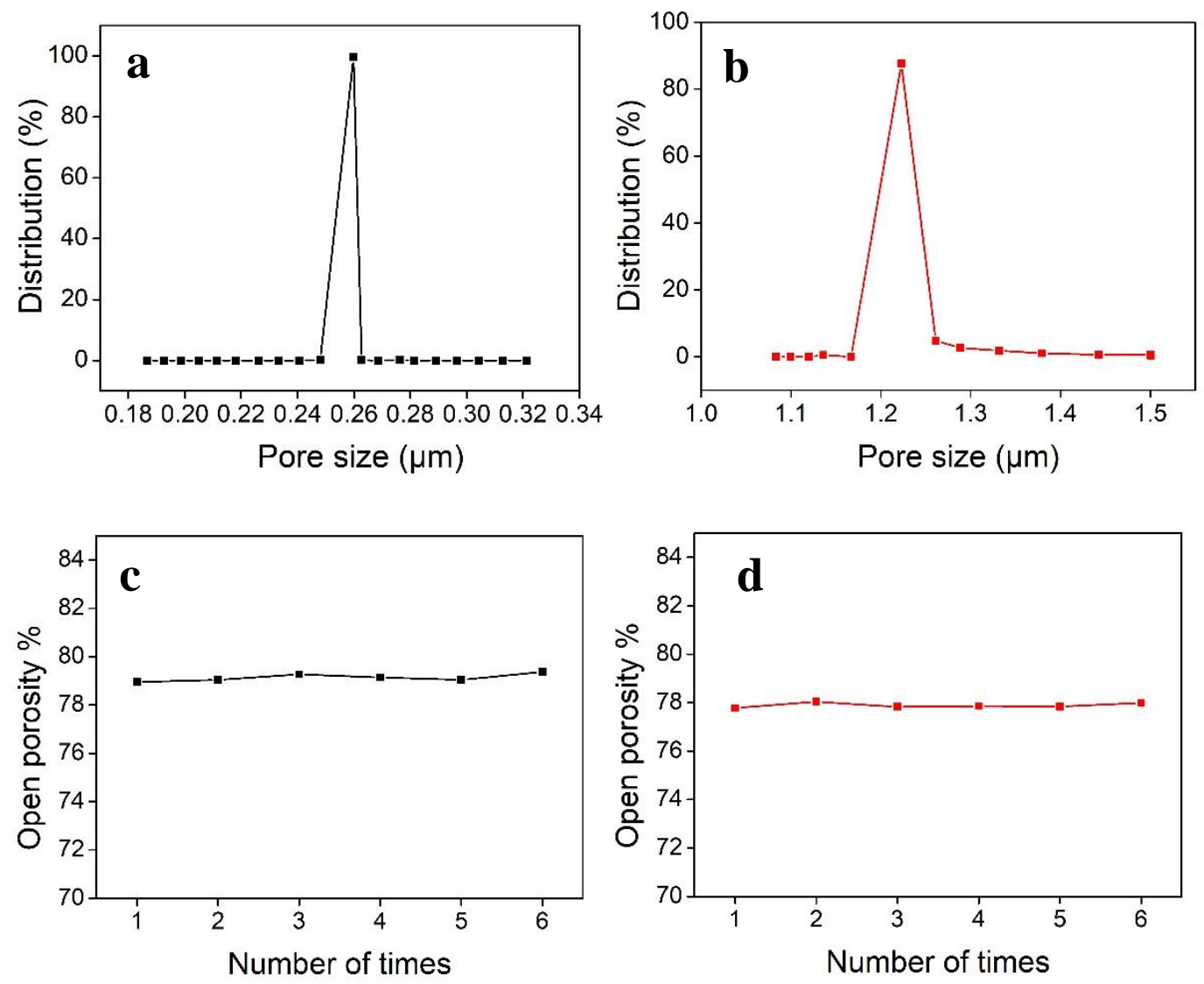

Figure S4 Distribution of pore size and open porosity data of PVDF membranes. a) Distribution of pore sizes of PVDF membranes with dense skin layers. Gas $\left(\mathrm{N}_{2}\right)$ permeance $3.16 \times 10^{-6}$ $\mathrm{m}^{3} /\left(\mathrm{m}^{2} \cdot \mathrm{pa} \cdot \mathrm{s}\right)$, Gas flux $1.09 \times 10^{2} \mathrm{~m}^{3} /\left(\mathrm{m}^{2} \cdot \mathrm{h}\right)(\Delta \mathrm{P}=0.1000 \mathrm{bar})$. b) Distribution of pore sizes of PVDF membranes without dense skin layer. Gas $\left(\mathrm{N}_{2}\right)$ permeance $6.81 \times 10^{-6} \mathrm{~m}^{3} /\left(\mathrm{m}^{2} \cdot \mathrm{pa} \cdot \mathrm{s}\right)$, Gas flux $2.46 \times 10^{2} \mathrm{~m}^{3} /\left(\mathrm{m}^{2} \cdot \mathrm{h}\right)(\Delta \mathrm{P}=0.1000 \mathrm{bar})$. c) Porosity of PVDF membranes with dense skin layer. d) Porosity of PVDF membranes without dense skin layer. Each type of sample was tested for 6 times. The average porosities of composite membranes with and without skin layer were $79.14 \%$ and $77.89 \%$, respectively. 

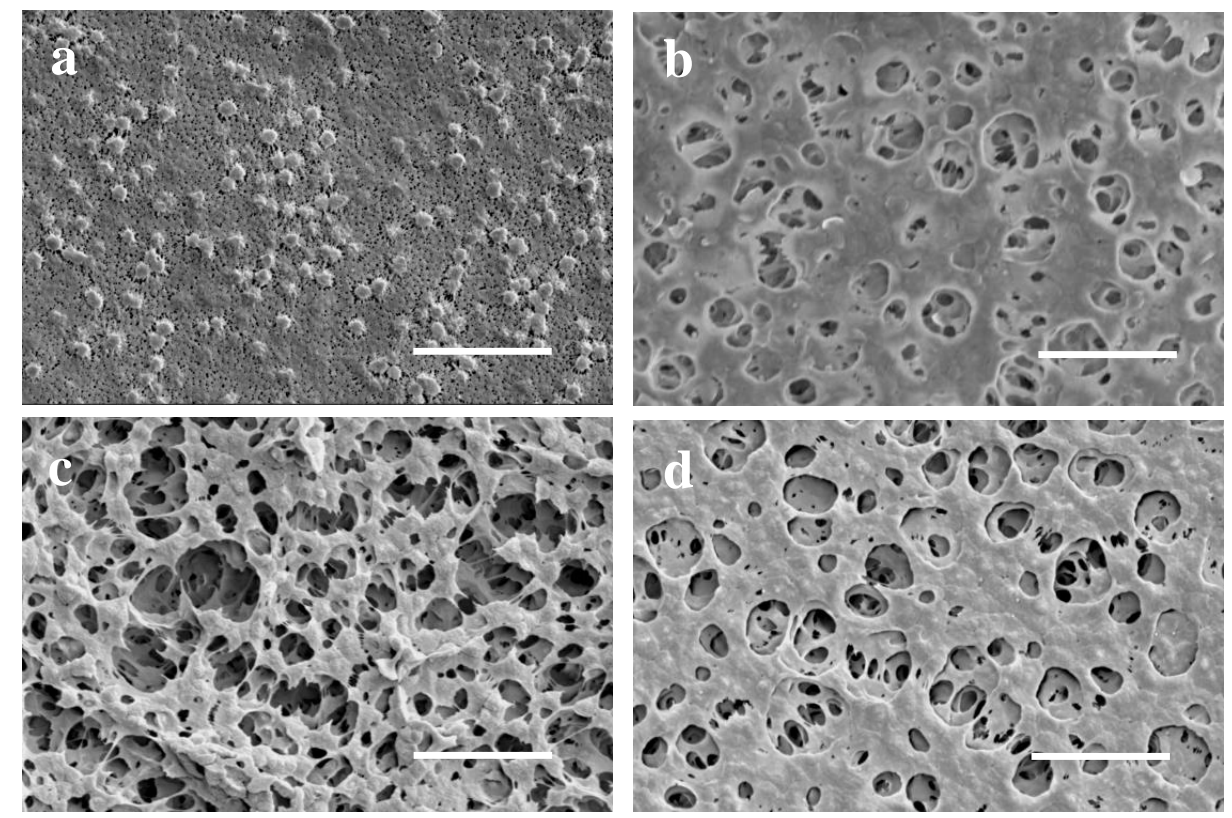

Figure S5 SEM images of PVDF membranes prepared by the VIPS method under different temperature and humidity conditions. a) $25{ }^{\circ} \mathrm{C}$, humidity $30 \%$; b) $60{ }^{\circ} \mathrm{C}$, humidity $60 \%$; c) $25{ }^{\circ} \mathrm{C}$, humidity $90 \%$; d) $60{ }^{\circ} \mathrm{C}$, humidity $90 \%$. The scale bar represents $10 \mu \mathrm{m}$. These results verified the adjustable property of pore size of PVDF membranes prepared by VIPS method. 

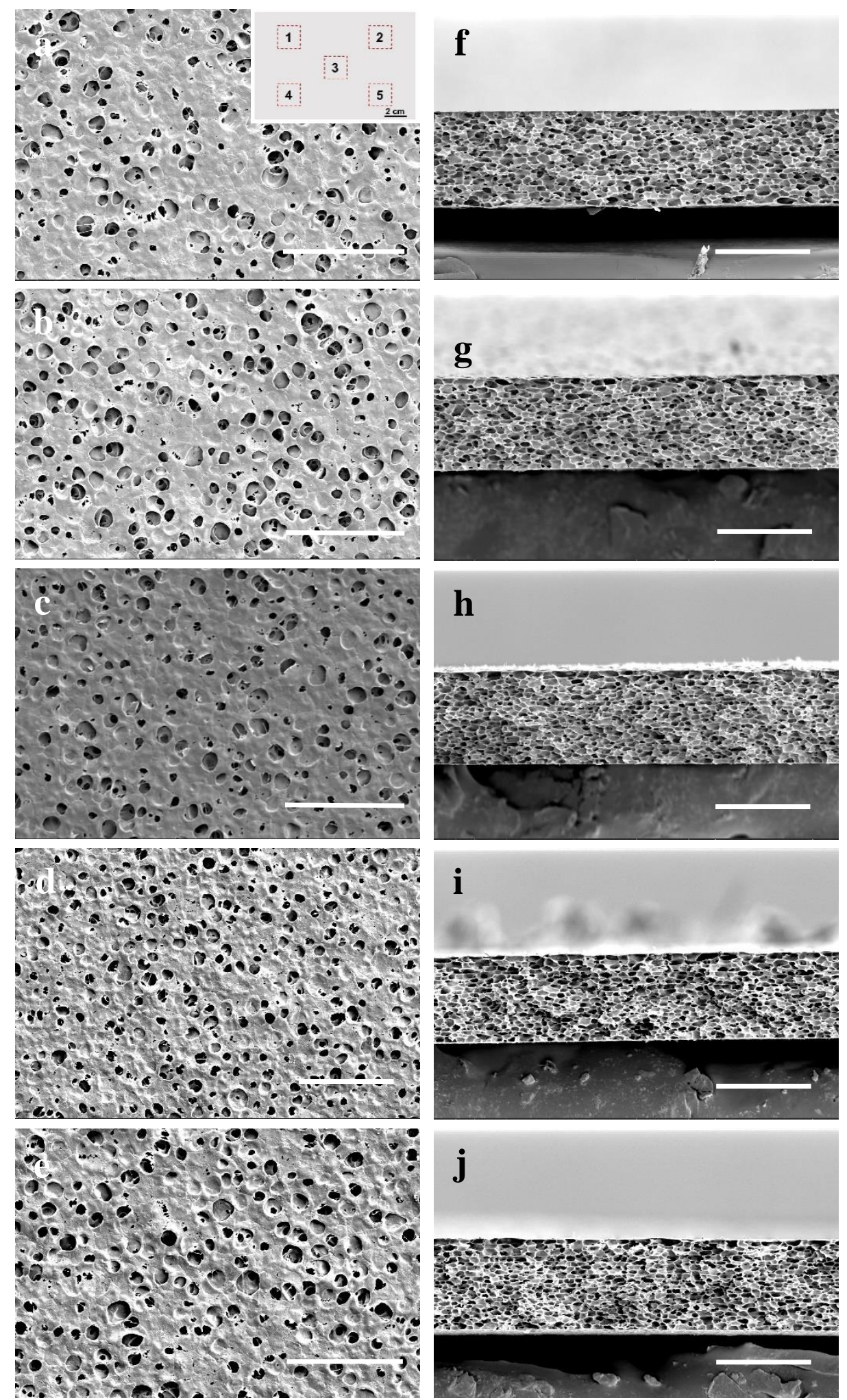

Figure S6 Homogeneity characterization of the decimeter-scale polymeric membranes. a-e) SEM images of samples cut from different parts of the decimeter-scale membranes (top view of the polymer substrate); inset in a is the schematic diagram of sample selection; SEM images a-e are corresponding to samples 1-5, respectively; f-j) SEM images of samples 1-5 (the corresponding section view). The scale bar represents $50 \mu \mathrm{m}$. 

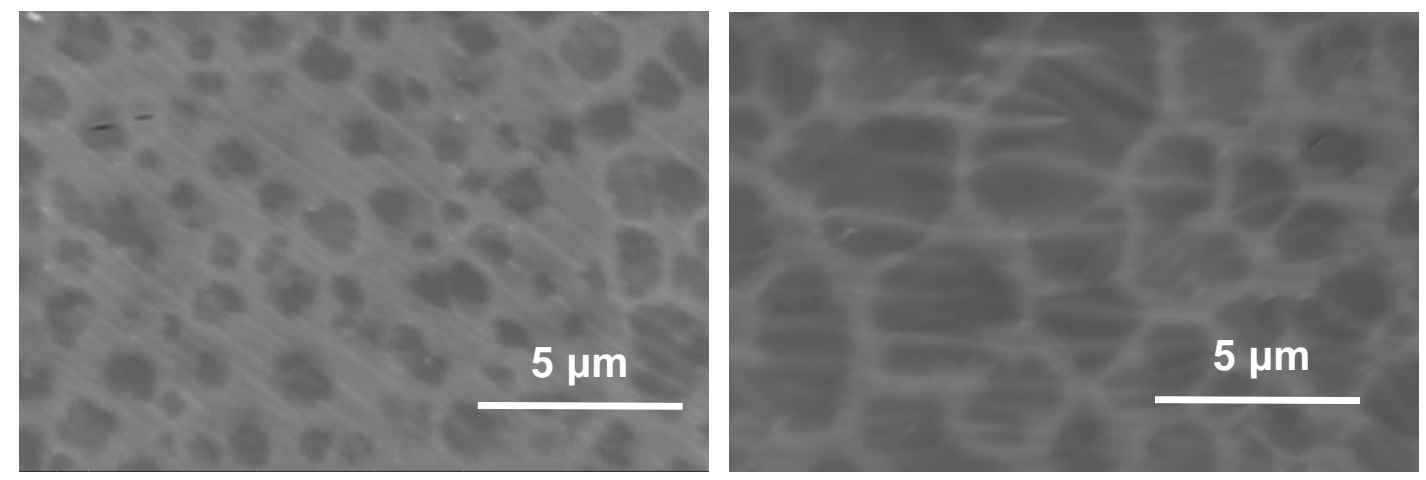

Figure S7 SEM images of the nanoporous atomically thin membranes (NATMs).

There are few defects generated during the transfer or sample preparation and SEM testing process. The existence of defects may cause the membrane failure during the $\mathrm{CO}_{2}$ transport test. The success rate of the NATMs was $\sim 90 \%$. For the defect originated during transfer process, it could be sealed by the interfacial polymerization method, which will be the next step of our work in the future. 


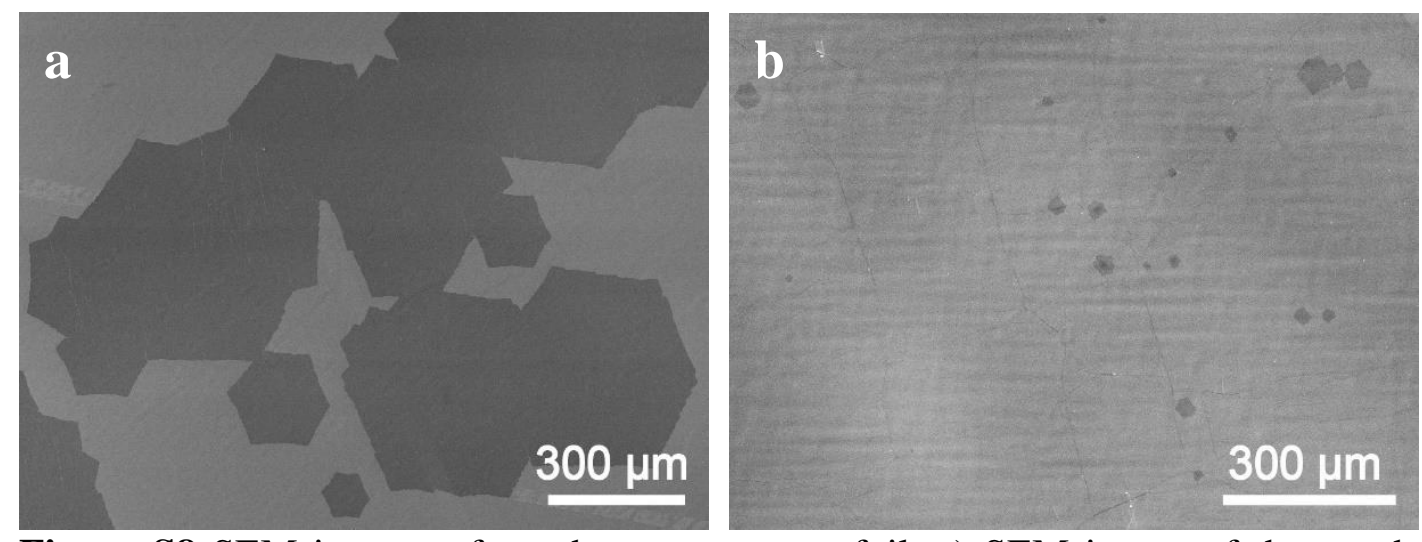

Figure S8 SEM images of graphene on copper foil. a) SEM image of the graphene before complete growth; b) SEM image of graphene after complete growth and full-covering the copper foil.

We can observe from Figure S8a that the graphene was polycrystal and the size of the crystal domain was $\sim 300 \mu \mathrm{m}$. 


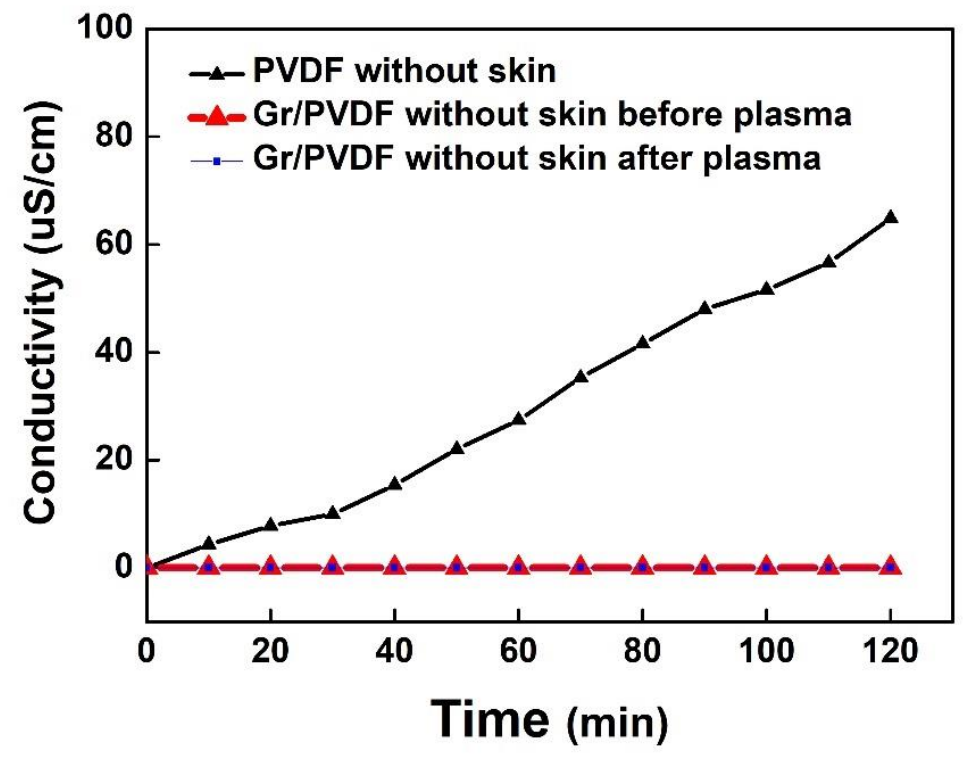

Figure S9 Diffusion-driven transport data of $\mathrm{KCl}$ through various membranes.

The pure PVDF membrane without skin layer produced by vapor-induced phase inversion method was salt-permeable; whereas the graphene/PVDF composite membranes before and after $\mathrm{O}_{2}$ plasma treatment were salt-resistant due to the existence of graphene films. 


\section{$1 \mathrm{~cm}$}

Figure S10 Digital photo of the nanoporous graphene/polymer composite membrane after separation experiment. The structure is intact, demonstrating the stability of NATMs during the diffusion-driven transport experiments. 